\title{
Seven deadly diseases rooted in your gut microbiome
}

\author{
Volume 5 Issue I - 2017 \\ jill C Carnahan \\ Flatiron Functional Medicine, USA
}

\author{
Correspondence: Jill C Carnahan, Flatiron Functional \\ Medicine, USA, Tel 303.993.7910, \\ Email DrJCarnahan@comcast.net
}

Received: August 22, 2016 | Published: January 03, 2017 role in human health and disease. From helping we absorb nutrients, to synthesizing vitamins and breaking down cellulose, we couldn't survive without this microbial zoo inside us.

Shortly after birth, we are colonized with over 1000 species of gut bacteria. Influences such as vaginal delivery vs. Cesarean section, breast-feeding vs. bottle feeding, antibiotic use, industrialized processed food, stress, levels, chronic illness and poor dietary choices all have a profound impact on which bugs predominate.

Recent data also shows that toxic chemicals, like glyphosate, may selectively destroy healthy microbes, leading to further imbalance and disease. Bacteroidetes and Firmicutes are the main bacteria in our gut which aid metabolization of undigested food remnants. Healthy bacteria in the gut promote the integrity of our gut lining, preventing "leaky gut" and thus prevent antigens and microbes from crossing over into the bloodstream. They also contribute to our host defense by regulating innate immune system. Intestinal dysbiosis (an imbalance in the gut microbes) leads to many deadly disease states, including inflammatory bowel disease, obesity, diabetes, liver disease, heart disease, autoimmunity, mood disorders, and even cancer.

\section{Did you know these seven deadly diseases may be rooted in abnormalities of your gut microbiome!?}

Inflammatory Bowel Disease: Inflammatory bowel disease is thought to be caused in part by aggressive T-cell mediated cytokine response to the commensal bacteria, which leads to chronic inflammation and damage of the intestinal lining. Another immune abnormality related to the development of Crohn's disease is that certain fighting cells, called macrophages, may have trouble recognizing which bacteria is friend or foe. They initiate an attack on what they perceive to be an enemy, when in fact it's just a harmless residential bacterium lining the gut. In a genetically susceptible people, inflammatory bowel disease may be related to this out of control response to commercial organisms. In addition, Seksik et al. found that the fecal microflora in patients with Crohn's disease contined significantly more enterobacteria than in healthy subjects.

In Ulcerative colitis (UC) the disease is limited to the distal colon. Studies have shown lower numbers of lactobacilli during the active phase of ulcerative colitis. Species, such as Lactobacillus salivarus, Lactobacillus manihotivorans and Pediococcus acidilactici were present in remission, but not in patients who had active inflammation of ulcerative colitis.

Obesity: Normal gut bacteria play an important role in development of obesity. ${ }^{1-3}$ Patients on a high-fat diet have higher levels

of Firmicutes and Proteobacteria and lower levels of Bacteroidetes, indicating that obesity may be associated with decreased diversity of species. The ratio of Firmicutes to Bacteroidesis higher in obese people and has been found to be correlated to body weight. Clostridium difficile infections may be another possible cause of obesity. Overweight individuals have more short chain fatty acids (SCFAs) than lean individuals, especially a particular SCFA, called propionate.

Certain bacteria (Bifidobacterium breve, Bifidobacterium bifidum and Bifidobacterium pseudolongum) were able to produce Conjugated linoleum acid (CLA) in the gut. CLA may have an antiobesity effect in humans. Another study showed that after eight weeks of feeding, L. rhamnosus PL60 reduced the body weight of obese mice without reducing energy intake. Prebiotics and probiotics can also be used to fight obesity. Besides probiotics, certain gut bacteria could also protect humans from obesity. The Bacteroidetes phylum, particularly Bacteroides spp., has been suggested to protect against increase weight gain.

Fecal microbiota transplantation (FMT) may also hold promise in treating obesity. Animals who received FMT from the obese twin donors developed increased body mass and obesity compared to those receiving transplants from the lean twin donors.

Diabetes: Type 1 diabetes is a destructive autoimmune disease attacking the pancreas islet cells causing the inability to produce insulin. Healthy balance of gut bacteria may reduce pro-inflammatory cytokines to protect patients against the development of Type 1 Diabetes. These changes helped to restore intestinal mucosal barrier function. Impaired intestinal mucosal barrier, also known as "leaky gut" and altered mucosal immunity are involved in development of this disease, and many other autoimmune diseases. Abnormal gut microbes may contribute to the development of type 1 diabetes, since cross-talk between gut bacteria and the innate immune system is one of the triggers for destruction of the islet cells of the pancreas.

Higher levels of Bifidobacterium species decreased the effects of endotoxemia. Endotoxemia is a very pro-inflammatory process 
that occurs when bacterial coatings, called LPS, cross over into the immune system through a permeable intestinal lining. There is perhaps no more pro-inflammatory trigger to disease than LPS, which has been linked to autoimmunity, depression, diabetes, heart disease and more. Endotoxemia is clearly associated with diabetes and obesity. Bifidobacterium was associated with improved glucose tolerance, improved insulin secretion and decreased inflammation.

Liver disease: The gut and liver work closely together. The liver receives approximately $70 \%$ of its blood supply from the intestinal venous outflow. The liver is the first line of defense against gutderived antigens and it is equipped with a broad array of immune cells; macrophages, lymphocytes, natural killer cells to accomplish this function. Gut bacteria play a key role in the maintenance of a healthy liver. Ethanol, ammonia, and acetaldehyde produced by the intestinal microflora are metabolized by the liver and heavy loads of these chemicals may cause inflammation and toxic overload in this filter organ.

Small intestinal bacteria overgrowth (SIBO) may play a role in the development of nonalcoholic fatty liver disease. Small intestinal motility is decreased in the presence of small intestinal bacteria overgrowth (SIBO) in rats with liver disease. Interestingly, antibiotic treatment of SIBO may improve non-alcoholic fatty liver disease. In addition, gut-derived microbial toxins and other bacterial products may be cofactors in the development of alcoholic liver disease.

Chronic heart disease: Gut bacteria composition has a direct link with the risk of cardiovascular diseases. The gut bugs form trimethylamine (TMA) from dietary choline. TMA may increase risk of atherosclerosis. In chronic heart failure (CHF) patients invasive Escherichia coli were identified. Increase in intestinal permeability in chronic heart disease may trigger inflammation, contributing to further impairment in heart function.

Colonization with Lactobacillus brevis decreased intestinal permeability, whereas Escherichia coli, Klebsiella pneumonia, and Streptococcus viridians showed the opposite effect. Increased intestinal permeability, or leaky gut, leads to bacteria translocation (endotoxemia) which is an important stimulus for inflammatory cytokines to be activated in chronic heart failure.

Cancer: It is well known that the presence of microbial pathogens or the disruption of the healthy microbiome may contribute to the development of gastrointestinal cancers, like stomach and colon cancers. It was reported that gut bacteria can trigger macrophages to produce products that damage DNA, inducing chromosomal instability in cells. Oxidative stress brought on by abnormal microbes may also contribute to inflammation in these cancers.

The composition of the gut bacteria is quite different between healthy individuals and colon cancer patients. Certain butyrateproducing bacteria (Ruminococcus spp. and Pseudobutyrivibrio ruminis) were low in the stool of colorectal cancer patients compared to healthy individuals. Butyrate is important for normal colon cell health. Two Prevotella species were completely absent from the colon cancer samples analyzed. Prevotella may help maximize energy from a plant-based diet. The higher levels of Prevotella in the healthy individuals may reflect differences in intake of fiber and plantfoods compared to the individuals with colon cancer. On the other hand, species like Acidaminobacter, Phascolarctobacterium, Citrobacter farmer, and Akkermansia muciniphila were significantly higher in colon cancer patients.

Autoimmune diseases: There is a clear link to autoimmune diseases, like rheumatoid arthritis and the gut microbiome. In one study, joint inflammation did not develop in animal models that were germ-free. Rheumatic arthritis patients had significantly lower levels of Bifidobacteria and Bacteroides fragilis. In addition, increased LPS uptake through the gut lining is a risk factor for development of arthritis.

It's time to take charge of your gut!

So now you know how important it is to keep your microbiome happy in order to prevent serious disease... It literally could mean the difference between life and death. A healthy microbiome starts with a whole food organic diet rich in plant foods, healthy fats, like coconut, avocado and olive oils, prebiotic fiber sources, nuts and seeds, probiotic-rich living foods, and a variety of colorful fruits and vegetables rich in phytonutrients and antioxidants.

\section{Acknowledgments}

None.

\section{Conflicts of interest}

Author declares there are no conflicts of interest.

\section{Funding}

None. 